\title{
Application of Rough Set Technology to Control Information System of CFBB
}

\author{
Mingming Gao ${ }^{1, \text { a }}$, Jie Hou ${ }^{1}$, Deliang Zeng ${ }^{1}$, Xiujian Lei ${ }^{2, b}$, Yuping $\mathrm{Wu}^{2}$ and \\ Mingsheng Zhang ${ }^{2}$
}

${ }^{1}$ Beijing Key Laboratory of Measurement and Control New Technology and System for Industrial Process, State Key Lab of Alternate Electric Power System With Renewable Energy Sources North China Electric Power University

China, 102206

\author{
${ }^{2}$ Sichuan Baima Circulating Fluidized Bed Exampled Power Limited Liability Company, \\ China, 641000 \\ a gmm1@ncepu.edu.cn, b gzjgao@tom.com
}

\begin{abstract}
Keywords: data mining, rough set, neural fuzzy inference, circulating fluidized bed boiler.
Abstract. Circulating fluidized bed boiler(CFBB) is a distributed parameter, nonlinear, time-varying and multivariate coupling system. Considering these, the core identity of Rough Set and the theory of self-organizing neural fuzzy inference system is applied to the control system of CFBB. The system is carried out toward the 300MW CFBB in Baima Sichuan province, and finally improve the performance of CFBB effectively.
\end{abstract}

\section{Introduction}

Technology of the circulating fluidized bed combustion attract serious attention because of its high efficiency, low pollution performance [1]. Its advantages are as follows: high combustion efficiency, wide adaptability to coal, fuel preparation system is simple, low temperature combustion to reduce emissions, furnace $\mathrm{NO}_{\mathrm{x}}$ desulphurization, load regulation performance. To alleviate the current energy shortage situation has the advantage be richly endowed by nature, since its inception has been widely used in the world. Compared with the conventional pulverized coal boiler, the dynamic process of CFB is very complex, many influence factors, has the strong coupling between the combustion system and steam system, object to such a distributed parameters, nonlinear, time-varying, multivariate coupling, the control system needs to control the task control system more complex than general boiler.

By analyzing the influence of various factors on the bed temperature, all have great effect on the bed temperature factors, thereby generating model of CFBB system mechanism [2]. In the formation process of CFB bed temperature system model in the past, which are based on the analysis of influence factors of the bed temperature and the coupling relationship between each parameter, according to the parameters of dynamic balance and energy balance equations to establish the relationship of phase zone, resulting in a circulating fluidized bed temperature can reflect the main characteristics of the system the relatively simple linear model. But this is the use of attributes reduction properties of the rough sets, to get the mechanism model.

Knowledge reduction is one of the core contents of rough set theory.In the knowledge system of S $=(\mathrm{U}, \mathrm{A})$, there are a lot of redundant attributes in A, while maintaining the same classification ability of condition, we can delete redundant attributes, this is the reduction of knowledge. If the $R$ in the $U$ on the indiscernibility relation ind $(R)=$ ind $(R-\{R\}), R \in R$, then $R$ is said to be simple. Let $S=<U$, $\mathrm{A}>$ be a decision-making system, among them, $\mathrm{C}$ is the condition attributes of decision attribute set, $\mathrm{D}=\{\mathrm{d}\}$. Then for any $\mathrm{a} \in \mathrm{C}$ attribute importance of SGF ( $\mathrm{a}, \mathrm{D})$ is defined[3,4].

$S G F(a, D)=H(D)-H(D \mid\{a\})$. 
That mutual information as attribute a and decision attribute of $\mathrm{D}$, its value is greater, the description property a to $\mathrm{D}$ more important decision. Let $\mathrm{U}$ be a field, $\mathrm{P}$ and $\mathrm{Q}$ as the two equivalence relations on cluster $U$, can think $U$ take an equivalence relation cluster is a random variable defined on $U$ subset consisting of, and can be determined by the following method and its probability distribution. Using K-means clustering method to obtain $\mathrm{P}$ and $\mathrm{Q}$ in the divided domain $\mathrm{U}$ for $\mathrm{X}$ and Y.

$$
\begin{aligned}
& X=\left\{X_{1}, X_{2}, \ldots, X_{n}\right\}, Y=\left\{Y_{1}, Y_{2}, \ldots, Y_{n}\right\}, \\
& (X: p)=\left(\begin{array}{cccc}
X_{1} & X_{2} & \cdots & X_{n} \\
p\left(X_{1}\right) & p\left(X_{2}\right) \cdots & p\left(X_{n}\right)
\end{array}\right) . \\
& (Y: p)=\left(\begin{array}{cccc}
Y_{1} & Y_{2} & \cdots & Y_{n} \\
p\left(Y_{1}\right) & p\left(Y_{2}\right) \cdots & p\left(Y_{n}\right)
\end{array}\right) . \\
& H(P)=-\sum_{i=1}^{n} p\left(X_{i}\right) \log \left(p\left(X_{i}\right)\right) . \\
& H(P \mid Q)=-\sum_{i=1}^{n} p\left(X_{i}\right) \sum_{j=1}^{m} p\left(Y_{j} \mid X_{i}\right) \log \left(p\left(Y_{j} \mid X_{i}\right)\right) .
\end{aligned}
$$

Usually, the bed temperature by the amount of fuel, one or two wind ratio, primary air, the amount of limestone, influence deslagging amount and flue gas recirculation quantity. In the process of simulation of the dynamic modeling system, often need to influence on various quantities are considered respectively, in order to simplify the system modeling, the combustion rate $\mathrm{Br}$ of this concept, comprehensively reflects the various parameters disturbance of the control volume.

$$
B r=\sum_{i=1}^{n} w_{i} X_{i}, i=1,2, \ldots, n \text {. }
$$

Wherein, control parameters coupling relationship for circulating fluidized bed boiler, for the influence of each parameter on the bed temperature change, the method is defined as follows.

$$
w_{i}=\frac{\frac{1}{S G F\left(X_{i}, T\right)}}{\sum_{i=1}^{n} \frac{1}{S G F\left(X_{i}, T\right)}}, i=1,2, \ldots, n .
$$

\section{Fuzzy neural network structure and the principle of organization control system}

Figure 1 is FNNCS (Fuzzy Neural Network Control System) that represents the neural fuzzy inference network controller, fuzzy neural network is actually a chapter in the simplified model, it directly to realize fuzzy, fuzzy rules fast parallel computing, and the average sentence, given the control quantity U, PNN said the index of network performance measurement, it represents the fuzzy performance measurement in organizational control query table, thereby correcting the control quantity[5,6]. Through the off-line training is the decision table PNN associative memory performance measurement, in order to provide FNNCS network on-line learning signals.

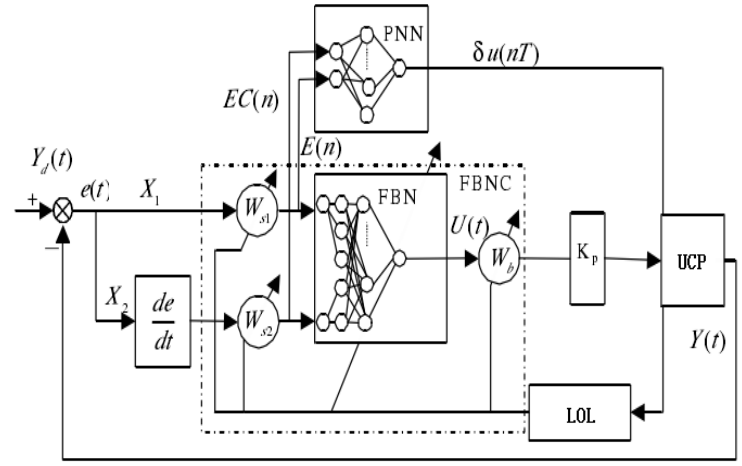


Fig. 1 Fuzzy neural network self organizing control system.

The network consists of 5 layers, the first layer is the input layer; the second layer is the fuzzy domain conversion factor layer, first need to determine, two parameters of the fuzzy control, because in the traditional fuzzy control, two parameters are adjusted by controlling the experience, in the vicinity of a working point of the system, so can not in a large range and good control results, the two parameters as the second layer of the network; third layer is the input membership function layer, here is the Gauss function; the fourth layer is the fuzzy rule layer; the fifth layer is the output layer.

\section{Fuzzy control in the simulation analysis and the results of CFBB}

The object is $300 \mathrm{MW}$ unit in power plant CFB boiler. Figure 2 shows a unit step load changes under the control of the disturbance for bed temperature perturbation. Boiler combustion rate is obtained using second section introduces knowledge. In the combustion rate under perturbation, the dynamic characteristics of bed temperature of pure delay can be approximated by two order inertial link a non minimum phase, according to Figure 2 income data, using the system identification method, get the combustion rate disturbance transfer function out of bed temperature response is as follows.

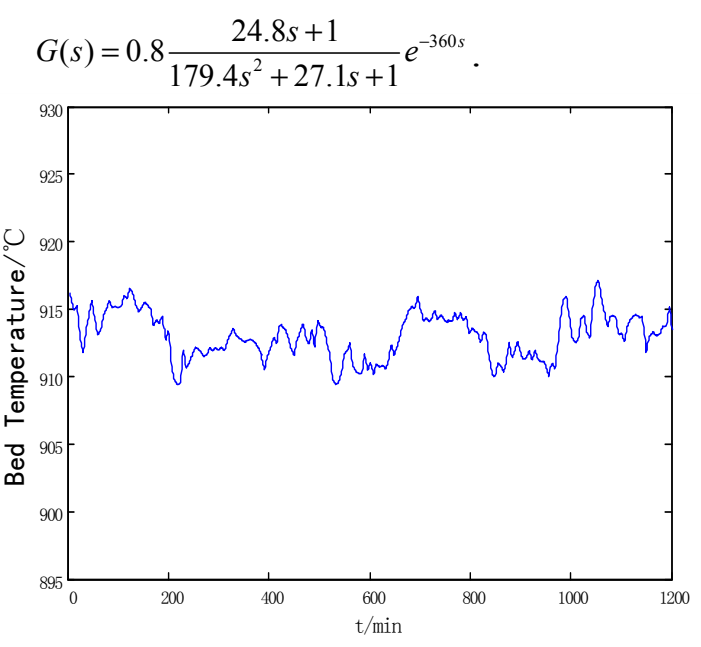

Fig. 2. Load step to variations of the bed temperature perturbations.

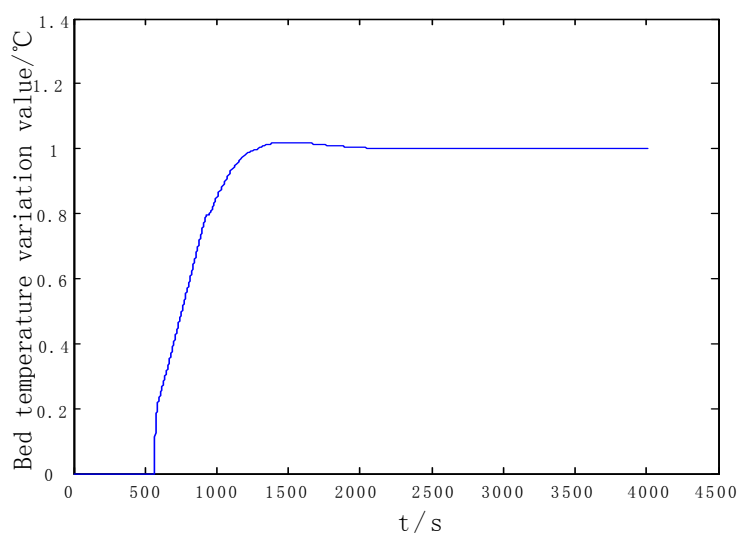

Fig. 3. The corresponding curves of traditional PI control.

The traditional PI control way is the response curve as shown in Figure 3. Input error of traditional PI control simulation system $\mathrm{E}$, input and output deviation change rate of $\mathrm{EC} \mathrm{u}$ for data cleaning, data generated as the fuzzy control rules, first of all to initialize network subtractive clustering is used to generate the original fuzzy controller, controller, membership function is shown in Figure 4. To train the fuzzy controller using BP neural network and error curve. The access controller trained to on-line control simulation control system and the control effect as shown in Figure 5. You can see the effect of fuzzy control, neural network control to the control effect is better than the traditional PI controller.

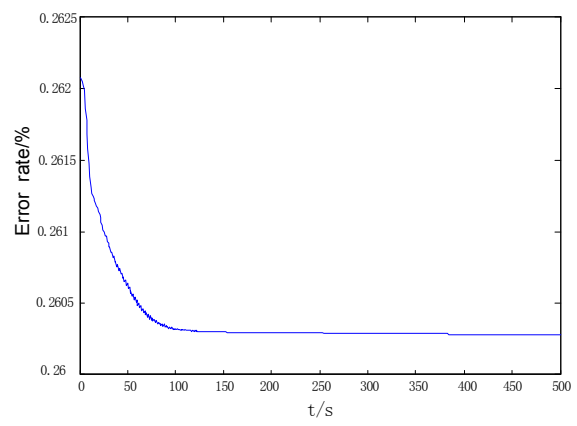

Fig. 4. The network training error curve.

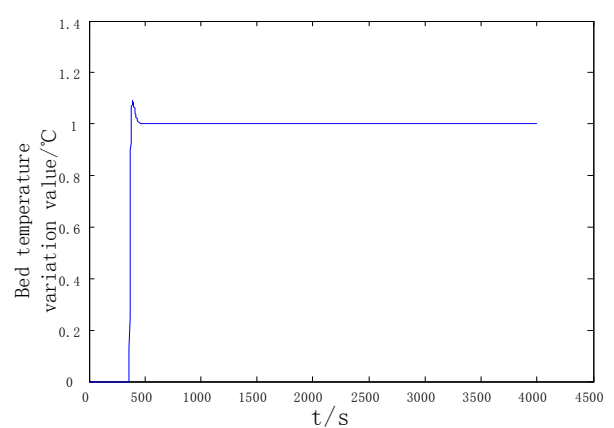

Fig. 5. The corresponding curves of fuzzy neural control system. 


\section{Summary}

Through data mining on the actual plant data, using rough set attribute reduction in the theory of the core properties, are all properties have great influence on the boiler bed temperature on the data processing of evolutionary, and get them to its influence degree, system identification model to generate the bed temperature. At the same time, the fuzzy neural self-organizing control strategy in the circulating fluidized bed boiler control system application, simulation research is carried out on the bed temperature control system, compared with the traditional PID, improves the efficiency of the circulating fluidized bed boiler.

\section{Acknowledgements}

This work is financially supported by the National Key Technology R\&D Program (2011BAA04B03), the National Natural Science Fund of China (51036002) and Sichuan Province Major scientific and technological achievements into the project funding (11CGZH0025). And the National High Technology Research and Development of China 863 Program (2007AA041105, 2007AA04Z163) and National Key Basic Research Program of China 973 (2012CB215203).

\section{References}

[1] Y.Z. Zhang, X.D. Xu, Dynamic mathematical model and Simulation of CFB boiler of, Chinese Journal of Electrical Engineering. Vol.20( 2000), pp. 84-88.

[2] J.S. Wang, R.Z. Zhang, Y. Zhang, Study of fuzzy neural network controller based on Rough Set, Journal of motor and control.Vol.12(2008), pp. 473-477.

[3] M. Zhou and L. F. Yan, Chinese Journal of Chemical Physics. Vol. 18 (2005), pp. 74-76

[4] K .Zhang, Journal of Fuel Chemistry and Technology. Vol. 31 (2005), pp. 75-79

[5] X. J .Li, W. H. Gui and Y. C. Zhang, Computer Measurement Control. Vol. 16 (2008), pp. 79-82

[6] S .Y. Hin, Harbin Industry of Technology. Vol. 2 (1996), pp. 91-93 